\title{
Toward Validation of Freeway Loop Detector Speed Measurements Using Transit Probe Data
}

\author{
Ahmed M. El-Geneidy and Robert L. Bertini
}

\begin{abstract}
Inductive loop detectors are used throughout the world to report individual and average vehicle speeds along freeway and arterial segments. Most loop detector data are aggregated over an arbitrary period such as 30 seconds, 1 minute or 15 minutes. Loop detectors are usually installed at locations convenient for operating an advanced traffic management system. It is very important to find the optimal spatial resolution for loop detector placement and the optimal temporal resolution for detector data reporting. The objective of this study is to examine this question at one site using a combination of loop detector and automatic vehicle location (AVL) data from a bus fleet. The bus fleet in Portland, Oregon is $100 \%$ automatic vehicle location equipped with certain buses running on express routes along freeways with inductive loop detectors. This study uses the AVL data to report speed at several spatial and temporal resolutions. These speeds are compared to speeds reported by inductive loop detectors. The study revealed the potential to improve speed data reported by inductive loop detectors by using the median speed reported by the detectors to represent segment speed during each temporal window. Further research is needed to further develop optimal strategies for loop detector placement and data aggregation/reporting.
\end{abstract}

\section{INTRODUCTION}

TNTELLIGENT transportation systems (ITS) are the application of advanced telecommunication and information technologies in order to enhance mobility and increase safety. Integration of data from different sources can lead to substantial benefits for system managers and users alike. The spatial and temporal resolution of the data that are being integrated often needs to be addressed when merging data from multiple sources. This study integrates Automatic Vehicle Location (AVL) data with inductive loop detector (ILD) data to capture the spatial and temporal

Manuscript received April 1, 2004. This work was supported in part by the Great Cities' Universities Coalition, Transportation Initiative Theme 7 Prototype for Advanced Public Transit Systems in Multimodal Corridors.

Ahmed M. El-Geneidy, is with the Center for Urban Studies, $506 \mathrm{SW}$ Mill Street Suite 350 Portland, OR 97201 USA (phone: 503-725-5946; fax: 503-725-8480; e-mail: elgeneid@ pdx.edu)

Robert L. Bertini is with the Department of Civil \& Environmental Engineering, Portland State University, P.O. Box 751, Portland, OR 97207-0751 USA (phone: 503-725-4249; fax: 503-725-5950; e-mail: bertini@pdx.edu). resolution of loop detector data that report average speeds along a freeway segment. This is accomplished through comparing the speeds reported by ILDs and with average segment speed recorded from AVL.

\section{Problem Statement}

Despite their more advanced capabilities, most ILDs are point-based sensors that are pre-programmed to report aggregate measures at predefined time intervals, including vehicle count, time mean speed and occupancy [1-3]. Point-based speed data are usually extrapolated over freeway segments defined by the midpoints between ILD locations. This extrapolation is used to assign a speed estimate to the entire freeway, which can be used to estimate travel times, delay and other performance measures for larger freeway corridors [4]. These measures are critical to the planning and operation of any transportation system. Clearly the transformation of a point-based parameter to report conditions over an inhomogeneous segment carry with it the potential for error-but the magnitude of this error is not wellunderstood.

ILDs have been installed on many freeways in United States and are typically used as fundamental data sources by advanced traffic management and information systems (ATMIS). Freeway speeds reported by ILDs are used for incident detection and for reporting real-time traffic conditions via various media, including web-based condition maps available in many cities. Displaying speed along segments was found to be more acceptable by public rather than point-based displays [5]. Yet the public perception changed after knowing that the values were extrapolated and might be misleading.

As part of ATMIS implementation, monitoring and improvement, it is important to examine the reliability of the system infrastructure and in most cases the loop detectors themselves. As more regions are developing archived data user services (ADUS) it is important to be able to determine how reliably the ILDs report vehicle speed. In general it is best to retain ILD data in their most raw form, e.g., at the lowest level of aggregation possible for archiving purposes. In many systems, the ILD data are available at 20- or 30-second levels of aggregation. But it is 
important to determine whether ILD data should be aggregated over longer temporal windows for reporting purposes. Some previous studies have attempted to estimate an optimal temporal window for reporting ILD speeds [6, 7], and in several cases authors chose five minute intervals as appropriate for capturing peak hour traffic conditions along freeways.

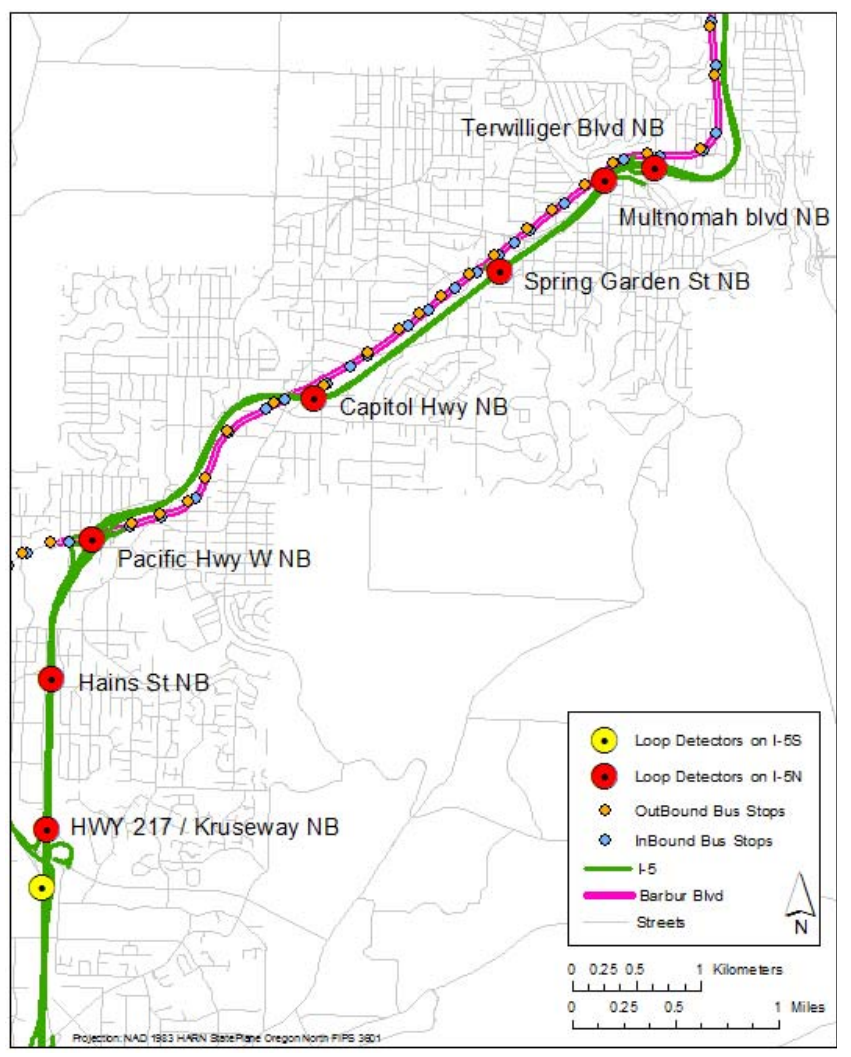

Fig. 1. Study corridor.

\section{CASE Study}

\section{A. Test Bed}

As shown in Figure 1, a freeway test bed was chosen in Portland Oregon that includes infrastructure-based and probe vehicle-based ITS data sources. The Interstate 5 corridor south of downtown Portland, Oregon, includes a heavily traveled freeway equipped with ILDs and with express bus operations. A parallel corridor is present (Barbur Blvd) and since the study concentrates only at reported speed from loop detectors this corridor will be considered in future research.

\section{B. Transit Data}

The Portland Tri-County Metropolitan Transportation District of Oregon (TriMet) operates an express bus service in the Interstate 5 corridor. Route 96X runs 31 times per day between downtown Portland and the communities of
Tualatin/ Sherwood. The route begins in downtown Portland with stops spaced at regular intervals. The route then traverses the I-5 freeway (with no stops) until it exits the freeway in Tualatin/Sherwood where it again resumes regular bus service with relatively frequent stops in this area.

TriMet's bus dispatch system (BDS) collects stop-level data as a part of an overall service control and management system. The BDS includes an AVL system based on global positioning systems (GPS) and a cellular/radio communications system enabling near real time dispatching [8].

Of relevance for this study, TriMet also archives stoplevel BDS data that are collected during the day for later off-line analysis. Each time a bus reaches a geo-coded stop, a new database entry is made on the vehicle's data card. These cards are removed at the end of each day for processing. The stop-level record includes an arrive time and leave time for each stop, whether or not the bus actually stops to serve passengers. When the bus does serve passengers, the dwell time and the numbers of boarding and alighting passengers are also recorded automatically using infrared-based automatic passenger counters (APCs) [9].

Normally the archived BDS data from Route 96X do not include any data along the I-5 freeway since database entries are only made at geo-coded stops where passengers are served. Since there are no stops along the freeway, database entries would normally only be recorded at the last stop before the bus enters the freeway and at the first stop after the bus exists the freeway.

For this experiment, TriMet coded a set of pseudo stops at pre-defined locations along the freeway. This provides time-stamped location data along the freeway for every bus trip using the freeway during the experiment. For this experiment, 18 pseudo stops were added in the northbound direction and 22 pseudo stops were added in the southbound direction. As shown in Figure 2, pseudo stops were located coincident with freeway ILD locations, at major freeway geometric inhomogeneities, and at the beginning and end of the study corridor. Figure 2 also shows that the study corridor contains six ILD segments and fourteen segments inscribed by pseudo stops.

\section{Freeway Data}

The Portland ILD system is programmed to report count, occupancy and time mean speed in each lane every 20 seconds. The Oregon Department of Transportation (ODOT) archives their data aggregated at a 15-minute level every day. For this experiment, the 20-second ILD data were archived between November 3 and November 24, 2002. This timeframe coincided with the period when TriMet coded the freeway pseudo stops. 


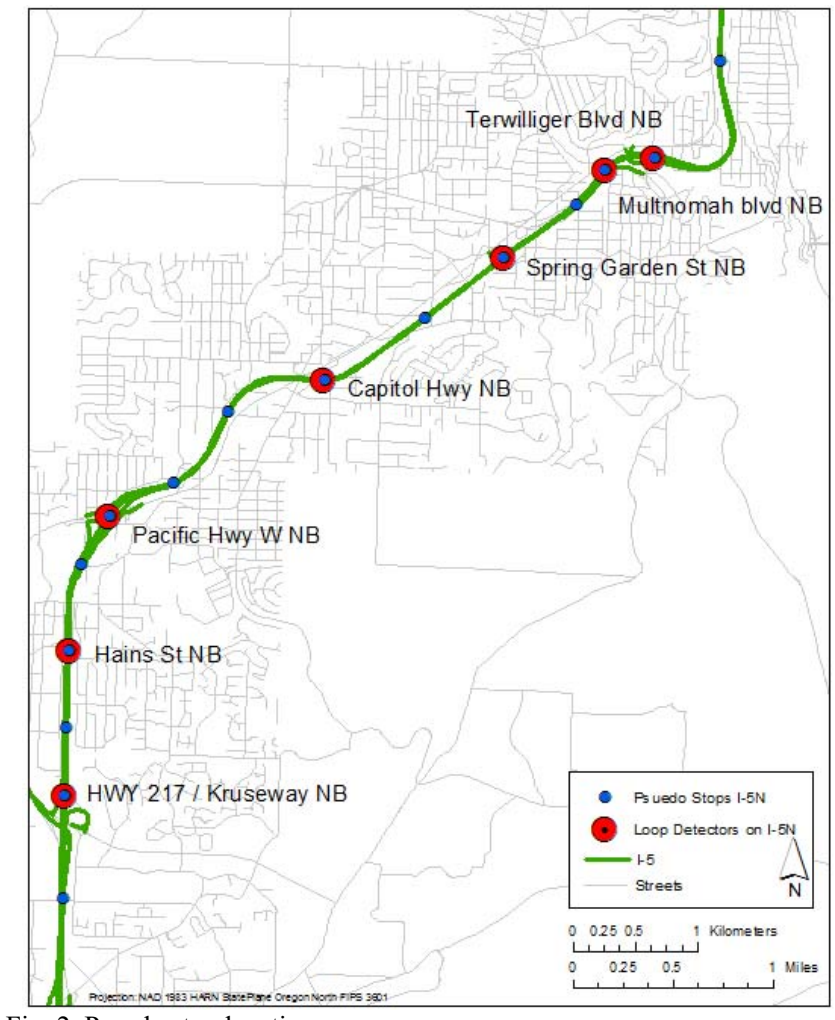

Fig. 2. Pseudo stop locations

\section{Probe Vehicle Data}

GPS-equipped probe vehicles were also dispatched along the freeway and the arterial on several days during the experiment to collect travel time data.

For this paper, we have selected one weekday (November 18, 2002) as a case study to focus on the examination of the consistency of freeway speed as measured by the infrastructure-based and probe vehicle based data sources.

\section{VALIDATION}

The distance measurements reported by the TriMet BDS were compared to distance measurements recorded by the other probe vehicles. As shown in Table 1, the distance measured between two pseudo-stops (coinciding with ILD locations) was very similar. This indicates that the BDS data are reliable for the purposes of this study. Note that the milepost distances reported by ODOT for detector

TABLE I

COMPARISON OF DISTANCE MEASUREMENTS BETWEEN HIGHWAY 217 AND SW HAINES STREET

\begin{tabular}{cc}
\hline \hline & Location ID \\
& $11372-11374$ \\
\hline Milepost Distance & NA \\
GPS Data & 1168.4 miles \\
Bus AVL Data & 1169.3 miles \\
Difference & $0.9 \mathrm{~m}(0.2 \%)$ \\
\hline \hline
\end{tabular}

locations were not used in this study, because ODOT's spatial resolution is too high for validating the BDS data (two decimal places).

\section{Comparing Measured Average Segment Speeds WITH ILD REPORTED SPEEDS}

In this section we examine the differences between average segment speeds measured by the bus AVL system and point speeds reported by the ILD system. Three scenarios are described below:

1) Comparing measured average segment speed with ILD reported speed using the nearest 20 -second interval;

2) Comparing measured average segment speed with a 5minute average ILD reported speed;

3) Comparing measured average segment speed with a 5minute median ILD reported speed.

Figure 3 shows a schematic of a freeway segment with the pseudo stop locations.

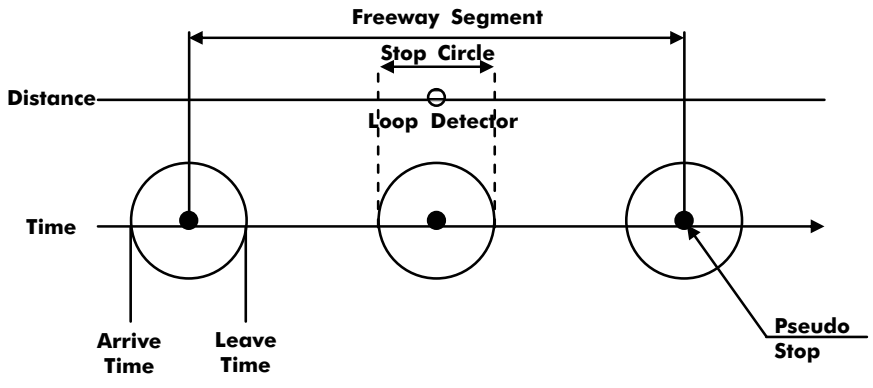

Fig. 3. Spatial and temporal resolutions

\section{A. Comparing measured average segment speed and 20-} second reported ILD speed

The average segment speed measured by the AVL system was calculated at pseudo stop $(x)$ by subtracting the time that the bus passed pseudo stop $(x+1)$ from the time it passed pseudo stop $(x-1)$ and dividing by the distance between pseudo stops $(x+1)$ and $(x-1)$. The location of pseudo stop $x$ was coincident with the location of an ILD. The following equation was used to estimate the segment speed using bus AVL data:

SegmentSpe ed $=\left(\frac{\text { PatternDis }_{x+1}-\text { PatternDis }_{x-1}}{\frac{\text { eave }_{x+1}+\text { Arrive }_{x+1}}{2}-\frac{\text { Leave }_{x-1}+\text { Arrive }_{x-1}}{2}}\right) *(60 * 60)$ Miles / hour

Note that PatternDist is the distance between any stop along a bus route and the first stop at the begging of the route. The approximate time that the bus passed over the ILD at the middle of the segment was estimated using Equation (2):

Time $=\frac{\text { Leave }+ \text { Arrive }}{2}$

This assumes that the bus was traveling at a uniform 
speed over the entire segment. The measured segment speed estimated from Equation (1) was matched with the reported ILD point speed during the 20 -second time interval coinciding with the time approximate time that the bus passed over the ILD (from Equation 2). The data were matched from both sources and a scatter plot is shown in Figure 4. Note that the speed is measured in miles per hour $(\mathrm{M} / \mathrm{H})$.

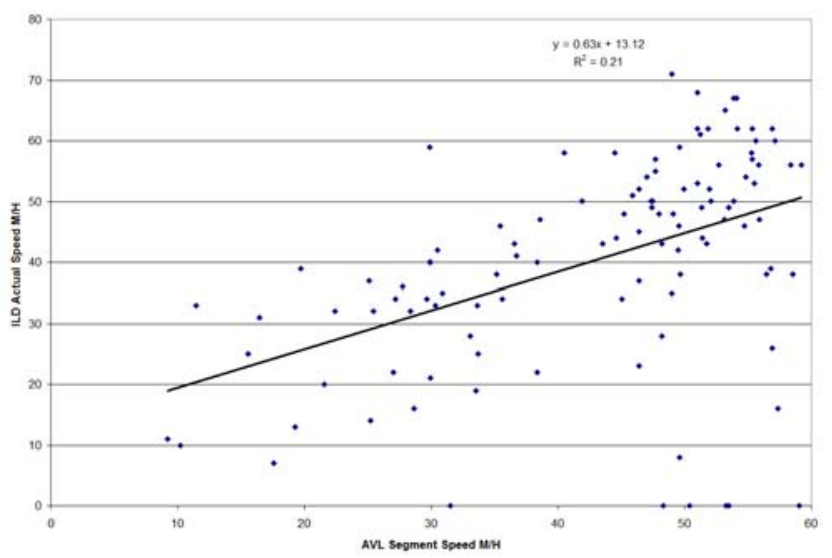

Fig. 4. Measured average segment speeds vs. 20-sec ILD point speeds.

In Figure 4, the measured average segment velocity forms the $x$ axis and the 20-second ILD reported point speed is on the $y$ axis. The best fit line (while expected to be a $45^{\circ}$ angle) is slightly flatter, with a relatively poor fit. The poor temporal resolution contributes to the measurement error because we do not know exactly when the bus was over the detector. This is because AVL data are recorded based on GPS settings.

\section{B. Comparing measured average segment speed and five-minute average ILD reported speed}

Next, we increased the temporal window for the ILD reported speeds to a five-minute average speed centered on the approximate time that the bus was located over the loop detector. Figure 5 shows the relationship between these two speed estimates using the measured average segment speed as the $x$ axis and the five minute average ILD reported speed as the $y$ axis. The relationship between the two parameters appears to be improved. Other temporal windows were tested, yet none of them had better accuracy than the five minute interval. This agrees with some previous findings [6].

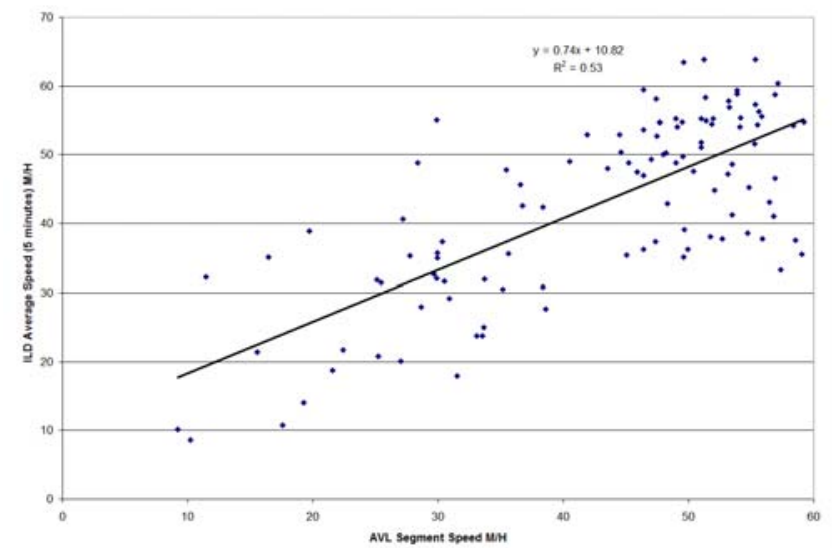

Fig. 5. Measured average segment speeds vs. five-minute ILD point speeds.

As an example, the five minute level of aggregation appears to fit the AVL data better than the 20 -second ILD data. An error term still exist in reporting the segment speed. It should be noted that there are similar problems with extrapolating the average travel time reported by the AVL system since we know that the bus does not really travel at a perfectly uniform speed along the segment.

\section{Comparing measured average segment speed with a} five-minute median ILD reported speed

To further explore the relation between speed measured via the AVL system and speeds reported by the ILD, a third comparison was made using the five-minute median ILD speed rather than the five-minute mean speed. From previous analyses, we know that the ILD contain some intervals with error codes or speed reported as zero (coinciding with intervals during which the count was zero). In order to alleviate some of the effects of this variability, it was hypothesized that the five-minute median might be more robust measure. Figure 6 shows the relationship between these two speeds using the measured average segment speed as the $x$ axis and the five-minute median ILD reported speed. This comparison contained the best fit relation of the three. 


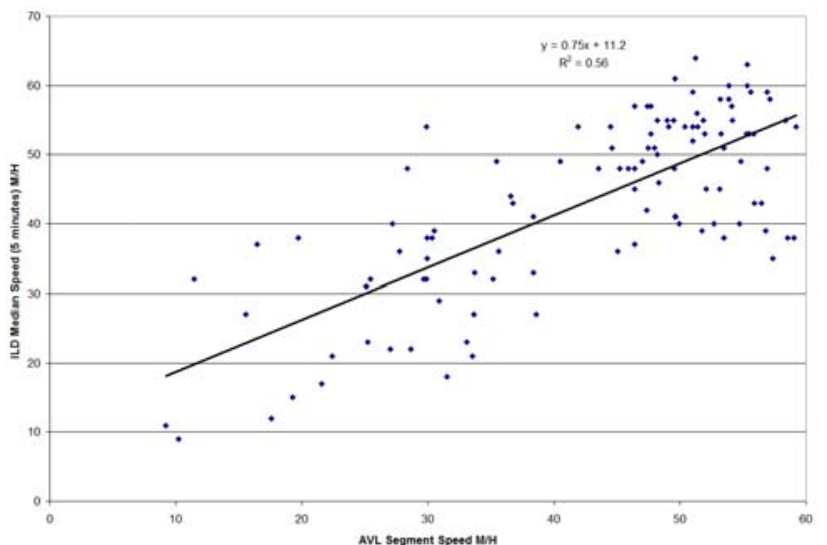

Fig. 6. Measured average segment speeds vs. five-minute median ILD point speeds.

A better estimate with lower measurement error is present when using the median speed measured over a five minute interval.

\section{Statistical ANALYSiS}

Currently we have three sets of matched data. Each data set included 110 observations. To examine the difference between two population means a paired sample test is used to differentiate between the matched data types. This test is used to test whether the two matched pairs are the same. Table 2 shows a summary of the test for each set.

TABLE 2 STATISTICAL ANALYSIS

\begin{tabular}{cccc}
\hline \hline & Case A & Case B & Case C \\
\hline $\mathrm{SD}$ & 1.54 & 0.89 & 0.86 \\
$\mathrm{t}$ & 1.66 & -0.03 & -0.61 \\
& Fail & Fail & Fail \\
\hline Range & $30.3 \mathrm{M} / \mathrm{H}$ & $4.0 \mathrm{M} / \mathrm{H}$ & $1.68 \mathrm{M} / \mathrm{H}$ \\
\hline \hline
\end{tabular}

The analysis of the three pairs found that we could not reject the null hypothesis, confirming that they are different and that further research is needed to quantify which one is better at reporting the true segment speed. The difference range was calculated for the three paired samples. Case $\mathrm{C}$ has shown the lowest variation $(1.68 \mathrm{M} / \mathrm{H})$ in the difference compared to the other two samples $(30.31 \mathrm{M} / \mathrm{H}$ and 4 $\mathrm{M} / \mathrm{H})$.

Results from the means tests indicate that calibration factors may need to be applied in certain instances when extrapolating speed from ILDs to segments. The National Institute of Standards and Technology (NIST) recommends that calibration factors be applied to values derived from a particular instrument when systematic errors are known to exist [10]. This is particularly relevant in cases where an instrument cannot be calibrated or where an instrument has reached its maximum precision. This is the case here where ILDs have reached their maximum precision in reporting speed at the point level, yet when extrapolating it along segments an error term was present $(+/-30 \mathrm{M} / \mathrm{H})$. The temporal aggregation enabled more declination in the error term. Both error terms $(+/-4 \mathrm{M} / \mathrm{H}$ and $+/-1.68 \mathrm{M} / \mathrm{H})$ appear to be within acceptable ranges. However it should be noted that we do not know the actual speed that the bus was traveling as it passed over the ILDs.

\section{CONCLUSIONS AND RECOMMENDATIONS}

When integrating data from multiple sources, it is important to define the appropriate temporal and spatial windows. Based on this preliminary analysis, the speed reported by loop detectors every 20 seconds when displayed on a freeway map could be misleading when extrapolated over a long inhomogeneous segment. Of the several cases examined, the five minute median speed appeared to be the most representative of measured speed along a segment. The five minute average was also in the accepted range for reporting speed.

The use of AVL data measured at specific points over freeway segments has been beneficial for calibrating speeds reported by ILDs along the same freeway segment. In this preliminary analysis, the integration of AVL and ILD data facilitated a reduction in the measurement error associated with aggregation and extrapolation. This was the concern of some people surveyed in [3]; when they were told that segment speed was accompanied with error they preferred the point speed even though it may not have been as meaningful to their freeway trip.

The findings in this analysis can help the ongoing research in the area of travel time estimates. ILD are installed along many freeways and can be used with origin/destination data to estimate travel times.

In the Portland metropolitan region, the inductive loop detectors report speed every 20 seconds. These data reported at low spatial resolutions may not be adequate to characterize actual speed profiles experienced by vehicles traveling along the freeway segment. Based on this analysis, it may be possible that improved speed estimates can be derived from using a rolling calculation of median speeds, for example over a five-minute interval. More research along these lines is underway using larger sample size.

\section{ACKNOWLEDGMENT}

The authors thank Prof. James Strathman and Dr. Thomas Kimpel, Center for Urban Studies, for assistance with the experimental design. The authors also thank Rex Fischer, Courtney Villegas, and Steve Callas if TriMet; Dennis Mitchell, and Jack Marchant of ODOT; and Bill Kloos, Willie Rotich, and Cliff Bolling at the City of Portland for their cooperation and support. 


\section{REFERENCES}

[1] B. McQueen and J. McQueen, Intelligent Transportation Systems Architectures. Boston, MA: Artech House, 1999.

[2] L. A. Klein, Sensor Technologies and Data Requirements for ITS. Boston, MA: Artech House, 2001.

[3] B. R. Hellinga, "Improving Freeway Speed Estimates from SingleLoop Detectors," Journal of Transportation Engineering, vol. 128, pp. 58-67, 2002.

[4] K. F. Petty, P. Bickel, J. Jiang, M. Ostland, and R. J., "Accurate Estimation of Travel Times from Single-Loop Detectors," Transportation Research Part A, vol. 32, pp. 1-17, 1998.

[5] C. Plaisant and P. Bhamidipati, "Vehicle Speed Information Displays for Public Websites: A Survey of User Preferences," presented at Intelligent Transportation Systems America, Washington DC, 2000.

[6] B. J. Gajewski, S. M. Turner, W. L. Eisele, and C. H. Spiegelman, "Intelligent Transportation System Data Archiving: Statistical Techniques for Determining Optimal Aggregation Widths for Inductive Loop Detector Speed Data," Transportation Research Record, vol. 1719, pp. 85-93, 2000.

[7] R. E. Brydia, S. M. Turner, W. L. Eisele, and J. C. Liu, "Development of Intelligent Transportation System Data Management," Transportation Research Record, vol. 1625, pp. 124130, 1998.

[8] J. G. Strathman, K. J. Dueker, T. J. Kimpel, R. L. Gerhart, K. Turner, P. Taylor, S. Callas, D. Griffin, and J. Hopper, "Automated Bus Dispatching, Operations Control, and Service Reliability," Transportation Research Record, vol. 1666, pp. 28-36, 1999.

[9] R. L. Bertini and A. M. El-Geneidy, "Modeling Schedule Recovery Processes in Transit Operations for Bus Arrival Time Prediction," Journal of Transportation Engineering, vol. 130, pp. 56-67, 2004.

[10] National Institute of Standards and Technology, Handbook 44: Specifications, Tolerances, and Other Technical Requirements for Weighting and Measuring Devices. Washington DC, 1998. 